\title{
Le fonctionnement des anaphores dans les textes oraux et écrits en français d'enfants bilingues et monolingues
}

\author{
Nathalie Decool-Mercier \\ Linguistique, Didactique et Francophonie (EA 4305 Université de Rouen) \\ natdecool@yahoo.fr \\ Mehmet-Ali Akinci \\ Laboratoire Dynamique du Langage (UMR 5596 CNRS - Université Lumière Lyon 2) \\ Mehmet-Ali.Akinci@ish-lyon.cnrs.fr
}

\section{Introduction}

«L'anaphore se définit traditionnellement comme toute reprise d'un élément antérieur dans un texte » (Riegel, Pellat \& Rioul, 1994 : 610). Cette définition communément admise de l'anaphore cache les diverses problématiques en jeu dans le processus anaphorique. Comme le rappelle De Weck (1991), le terme «anaphore » recouvre une réalité différente selon le point de vue théorique adopté. Les études portant sur le fonctionnement des anaphores ont été nombreuses au cours des vingt dernières années (Fox, 1987 ; De Weck, 1991 ; Corblin, 1995, 2005 ; Hickmann, 2004, entre autres). Ces études concernaient cependant essentiellement la narration à partir d'un livre imagé. Dans son étude du développement des processus anaphoriques, De Weck (1991) montre que le fonctionnement anaphorique diffère en fonction de l'âge, mais aussi en fonction du type de texte. Elle note que «la maîtrise du fonctionnement anaphorique, observée dans les textes de type histoire, ne se généralise pas d'emblée aux textes discursifs. Plus précisément, à un même degré scolaire, on observe des décalages très nets entre les différents types de texte, certains n'étant même pas totalement maîtrisés à 14 ans, comme les textes argumentatifs » (1991 : 309).

Dans cet article, nous avons choisi d'étudier le fonctionnement des anaphores dans des textes narratifs et expositifs produits en français par des enfants bilingues franco-turcs âgés de 10 ans et par leurs pairs monolingues français. L'étude porte sur 48 sujets de CM2 scolarisés dans le même établissement scolaire et d'origine socio-économique défavorisée. Chaque sujet a produit quatre textes : un texte narratif oral, un texte narratif écrit, un texte expositif oral et un texte expositif écrit. Les bilingues ont produit leurs textes dans les deux langues. Le texte narratif concerne une expérience personnelle : le sujet devait raconter un conflit (bagarre ou dispute), et le texte expositif demandait d'exprimer une opinion sur la violence et les incivilités à l'école. Seuls les textes en français sont ici analysés. A travers le corpus, notre objectif est de répondre aux questions suivantes :

1) Les sujets monolingues et bilingues scolarisés au CM2 ont-ils les mêmes usages des anaphores ?

2) Existe-t-il une différence entre les types de textes narratif et expositif ?

3) La modalité orale ou écrite influence-t-elle l'usage des procédés anaphoriques?

Dans la première partie de cet article, nous faisons un état des lieux sur les différentes approches de la notion d'anaphore en nous focalisant particulièrement sur son fonctionnement en général et son usage au cours de l'enfance. La seconde partie présente la méthodologie utilisée : les sujets, les procédures de collecte et de transcription des données. Dans la troisième partie, nous exposons les résultats les plus significatifs des analyses comparatives portant sur la densité des anaphores, ainsi que leur distribution quantitative et qualitative. 


\section{Différentes approches de la notion d'anaphore}

Pour la grammaire traditionnelle, la catégorie anaphorique ne peut être abordée que par l'étude des définitions des pronoms et déterminants. Cependant, cette étude ne mène pas à une vision unifiée des anaphores puisque la langue n'est pas ici considérée comme système (au sens de la linguistique structurale) et que les parties du discours sont étudiées de façon juxtaposée.

La linguistique structurale, quant à elle, se donne pour objectif l'analyse du fonctionnement de la langue en tant que système. Ainsi, le distributionnalisme permet de doter la catégorie des anaphores de critères de classification plus stables. Les anaphores apparaissent dans la classe des substituts dont l'extension varie toutefois selon les auteurs, notamment pour ce qui concerne les pronoms et les déterminants. Dans le cadre de la grammaire générative et fonctionnelle, déterminants et pronoms sont traités dans le cadre de la syntaxe du nom. «La relation anaphorique correspond alors à la référence dans le discours de ses différentes extensions et peut se réaliser aussi bien au moyen des déterminants définis que des pronoms personnels de troisième personne, possessifs et démonstratifs » (De Weck, 1991 : 17).

Avec le fonctionnalisme apparaissent deux éléments importants : un lien de dépendance entre anaphore et relation du texte au contexte extralinguistique (ancrage) et restriction de la notion d'anaphore à certaines sous-catégories de monèmes pronominaux. Pour la valeur anaphorique, l'identification précise du référent est une condition de base. Dans le courant de la grammaire fonctionnelle, la fonction anaphorique n'est donc clairement thématisée que pour les pronoms, et cette fonction est distinguée de la valeur déictique en fonction de la nature de la référence (De Weck, 1991).

\subsection{Conception textuelle de l'anaphore}

La conception textuelle de l'anaphore retenue ici est celle définit par Corblin : «On parle d'anaphore lorsqu'un élément (...) exige d'être interprété par emprunt à un terme du contexte proche, lorsqu'il y a dépendance nécessaire d'un « anaphorique » à un antécédent qui se comporte comme source » (1995: 177), l'antécédent n'appartenant pas forcément à la même phrase. En effet, dans le cadre de l'étude de l'anaphore, la phrase ne paraît pas être une unité satisfaisante puisqu'il est fréquent dans un segment de discours que l'antécédent se situe en dehors de la phrase contenant l'élément anaphorique. De Weck (1991) précise la définition de l'anaphore en lui attribuant trois composantes :

- la dépendance co-textuelle qui signifie que l'anaphore doit être interprétée en fonction du texte dans lequel elle est insérée ;

- la présence d'une source identifiable dans la portion de texte qui précède, le terme de source mettant « l'accent sur l'idée d'une origine sémantique à partir de laquelle une chaîne anaphorique est créée » (De Weck, $1991: 36)$;

- la reprise de la source, mettant en évidence la continuité des éléments thématiques assurant au texte sa cohésion.

La relation anaphorique apparaît ainsi comme une relation orientée, l'anaphorisé (ou antécédent ou source) précédant l'élément anaphorique. Afin de bien préciser la notion d'anaphore, il convient de plus de la différencier de deux notions que sont la co-référence et la deixis.

\subsection{Anaphore, co-référence et deixis}

Distinguer anaphore, co-référence et deixis revient à poser le problème de la référence. Comme le rappelle Favart, «Les anaphores appartiennent à la catégorie des marques de référence » (2005: 5). Selon Halliday et Hasan « Il existe certains items dans toute langue qui ont la propriété de référence, dans le sens spécifique avec lequel nous employons ici le terme ; c'est-à-dire qu'au lieu d'être interprétés sémantiquement pour eux-mêmes, ils font référence à quelque chose pour leur interprétation » (1976 : $31)$. 
S'agissant de la structuration du champ des termes métalinguistiques, Kerbrat-Orecchioni (1999) distingue la référence relative (dans laquelle elle place l'anaphore) de la référence absolue : la référence relative exprime le fait que l'interprétation d'une séquence verbale ne peut se faire d'après son sens propre (cas de la référence absolue) mais nécessite le recours à des éléments complémentaires. Ces éléments peuvent se trouver dans le contexte de la situation d'énonciation ou dans le co-texte. Si on se réfère à Benveniste (1966), la situation d'énonciation correspond à «la mise en fonctionnement de la langue par un acte individuel d'utilisation » et comprend le locuteur, l'interlocuteur, le lieu d'échange et le moment de l'échange. Ces paramètres s'inscrivent dans la langue à travers la deixis c'est-à-dire « la localisation et l'identification des personnes, objets, processus, événements et activités (...) par rapport au contexte spatio-temporel créé et maintenu par l'acte d'énonciation» (Lyons, $1980: 261$ ). La référence déictique renvoie donc au cas où le référent se situe dans la situation de communication immédiate. Les unités linguistiques marquant ce référent dans la phrase sont appelées «déictiques » (Benveniste, 1966) ou «embrayeurs» (Jakobson, 1963). Halliday et Hasan emploient quant à eux le terme d'exophore lorsque l'élément présupposé ne peut être trouvé dans le texte mais dans le contexte de situation. Ils précisent que «la référence exophorique n'est pas cohésive puisqu'elle ne lie pas deux éléments dans un texte $\gg(1976: 18)$.

Pour ce qui concerne la référence relative au co-texte, c'est-à-dire la référence relative qui dépend du contexte linguistique, Kerbrat-Orecchioni (1999) distingue les termes relationnels (au nombre desquels les termes de parenté, certains adjectifs et certains verbes) des représentants, termes «qui reçoivent leur signification d'autres termes, expressions ou propositions contenus dans le même texte et qu'ils représentent » (Haroche \& Pêcheux, 1972: 17). C'est dans la catégorie des représentants que KerbratOrecchioni classe les anaphoriques qu'elle distingue des anticipants ou cataphoriques. On retrouve ici la distinction proposée par Halliday et Hasan (1976) : dans le cas où l'élément présupposé est antérieur à la présupposition, le terme d'anaphore sera utilisé. Toutefois, la présupposition peut prendre une autre direction, l'élément présupposé apparaissant alors dans la suite du texte. Halliday et Hasan désignent ce cas de relation cohésive sous le terme de cataphore.

Hickmann (2001) insiste quant à elle sur la notion d'ancrage. L'ancrage renvoie à l'indexalité du langage, c'est-à-dire que le sens d'une expression est lié au contexte d'énonciation (paramètres personnels et spatio-temporels). Elle distingue deux types d'emplois dans la composante indexicale: les emplois déictiques et les emplois intra-discursifs. Ainsi, les pronoms appartiennent au système référentiel en ce sens qu'ils peuvent renvoyer aux interlocuteurs ou se substituer aux noms.

La co-référence, enfin, correspond à l'identité de référence. Le cas le plus simple de co-référence consiste en la répétition du nom. Corblin rappelle que «l'identité si elle vaut n'est en rien déductible d'une règle interprétative stipulant qu'un terme est à interpréter localement par répétition » $(1995$ : 78).

\subsection{Types d'anaphores}

Si l'on retient le point de vue sémantique, on peut distinguer :

- l'anaphore fidèle : l'élément anaphorique est identique à l'antécédent sur le plan lexico-syntaxique, avec un simple changement de déterminant entre les deux occurrences. Exemple : Un enfant... Cet enfant...

- l'anaphore infidèle dans laquelle l'anaphorique est lexicalement différent de la source, avec là aussi un changement de déterminant entre les deux occurrences. Exemple : Un cheval... L'animal...

- l'anaphore associative : il n'y a plus ici co-référence, mais l'antécédent et l'anaphorique sont liés par une relation du type partie-tout ou méronymique. Exemple : Au loin, on apercevait une église. Le clocher était illuminé, les vitraux brillaient (Kleiber, $2001: 1$ ).

- l'anaphore "coréférentielle » : l'antécédent et l'anaphorique renvoient au même référent.

- l'anaphore divergente ou «indirecte » ou « in absentia » : antécédent et anaphorique ne renvoient pas aux mêmes référents. Exemple : J'ai oublié mon livre. Peux-tu me prêter le tien? 
- l'anaphore conceptuelle: l'antécédent est constitué d'un syntagme étendu ou d'une phrase et il est résumé par l'anaphorique. Exemple: L'élève ne cessait d'importuner ses camarades. Cette mauvaise conduite lui a valu une punition. Courtès (1991) insiste dans ce cas de figure sur les notions d'expansion et de condensation, l'anaphorisant reprenant sous forme condensée l'anaphorisé présenté initialement en expansion

La typologie retenue pour la présente étude est celle proposée par De Weck (1991), inspirée des travaux de M. Charolles (1978). Elle retient 12 types d'unités anaphoriques, réparties en trois catégories : deux principales, les pronoms et les syntagmes nominaux, et une catégorie résiduelle intitulée «autres" composée essentiellement d'adverbes de lieu. Nous présentons cette typologie de manière détaillée avec des exemples extraits de notre corpus dans le point 3 (méthodologie).

\subsection{Fonctionnement des unités anaphoriques}

D'après De Weck (1991), le fonctionnement des unités anaphoriques a été étudié de manière différente selon que les auteurs se sont intéressés au niveau phrastique ou au niveau textuel. Le but de telles études est de déterminer quelles sortes de contraintes (syntaxiques, sémantiques et/ou textuelles) président à l'emploi des anaphores. En d'autres termes, qu'est-ce qui motive le choix d'un type d'anaphore plutôt qu'un autre? Quelles valeurs prennent les anaphores dans un texte donné ? Comment sont gérées les ambiguïtés potentielles? Telles sont les questions auxquelles les chercheurs ont tenté de répondre.

Au niveau phrastique, les études ont plutôt porté sur les catégories d'unités, notamment sur les pronoms et les articles définis - opposés aux indéfinis. Dans ce dernier cas, les articles indéfinis sont utilisés dans l'item source, tandis que les pronoms, les articles définis et les démonstratifs sont employés dans la reprise de la source (Bronckart, 1997, Hickmann, 2001). Halliday et Hasan (1976) notent que lorsqu'une relation cohésive (comme l'est la relation anaphorique) survient à l'intérieur d'une phrase, la structure même de la phrase impose des restrictions quant à la forme que prendra la réalisation de la cohésion, notamment par les lois de pronominalisation. Pour définir ce phénomène, Corblin (1995) reprend la distinction introduite par Chomsky entre anaphore liée et anaphore libre, distinction redéfinie par Milner (1982). Pour ce dernier, l'anaphore libre, insensible aux contraintes du sujet et des phrases, ressortit au discours (le discours excédant selon lui les limites de la phrase), tandis que l'anaphore liée, dépendante des contraintes citées, « ressortit exclusivement à la phrase » $(1982: 363)$.

Au niveau textuel, l'apport des diverses recherches va être de dépasser le niveau purement local pour aborder les anaphores à un niveau global. Les anaphores vont ici être mises en jeu dans le maintien de la référence à l'intérieur du texte, notamment par le biais des chaînes référentielles (Chastain, 1975 ; Bronckart, 1996 ; Corblin, 1995, 2005).

\subsection{Développement de la cohésion et des anaphores}

La plus grande partie des études s'intéressant à la cohésion, à la cohérence et aux anaphores ont concerné des textes narratifs rédigés par des sujets adultes. De plus, la compréhension a davantage été étudiée que l'expression, ceci en raison des contraintes expérimentales plus complexes lors de la production (il est difficile de prévoir si la notion étudiée sera produite par le sujet dans son texte).

Les études expérimentales concernant ces domaines et menées auprès d'enfants dans une perspective longitudinale ont elles aussi tout d'abord concerné la compréhension. Toutefois, la capacité à produire un texte cohérent et cohésif est indispensable à la réussite scolaire. Des études ont donc été menées qui se sont intéressées à la production de textes oraux et/ou écrits par des enfants. Il s'agissait ici encore le plus souvent de textes narratifs produits à partir de supports imagés, tels que "Frog where are you? " (Mayer, 1969), histoire sans texte présentée sous forme d'un livret de 24 pages, qui présente l'avantage d'être utilisable pour des narrations réalisées par des locuteurs de langues différentes (Berman \& Slobin, 1994). En effet, le récit est considéré comme la structure idéale pour l'étude de la référence et de la thématisation, puisqu'il est basé sur l'évolution de personnages principaux ou secondaires tout au long de l'histoire (Favart, 2005). Selon Karmiloff et Karmiloff-Smith (2003 : 197), la cohérence et la cohésion 
jouent un rôle distinct dans le développement de la production et la compréhension du récit, mais elles sont intimement mêlées dans le développement langagier de l'enfant.

Bien que les prémices de l'anaphore puissent être pressentis dans les pronoms de deuxième personne dès avant 2 ans dans un contexte dialogique (Salazar-Orvig et al., 2004), les fonctions discursives du langage, impliquant la maîtrise du système référentiel à différents niveaux de contrôle ainsi que celle de la cohérence et de la cohésion, émergent tardivement vers 6-7 ans et continuent à évoluer jusqu'à 10 ans et même après cet âge (Hickmann, 2004)

De Weck (1991) montre elle aussi, à l'instar du modèle de Karmiloff-Smith (1996), que les enfants distinguent peu à peu le texte du contexte, comme en témoigne le passage d'un fonctionnement purement déictique à une prise de distance avec la situation de production. En plus des mécanismes déictiques, ils développent des mécanismes intratextuels puis créent des oppositions distinctives entre les anaphores.

Le développement de la cohésion anaphorique est mis en évidence par l'évolution de la constitution des chaînes anaphoriques (introduction d'un élément et maintien de sa référence) et d'autre part par l'évolution des choix des unités qui composent les chaînes de référence.

Dans le domaine du maintien de la référence, le statut des reprises apparaît dépendant du mode d'introduction : les reprises non anaphoriques sont nettement prédominantes chez les plus jeunes, tandis que les reprises anaphoriques deviennent majoritaires vers 8-9 ans, avec stabilisation du point de vue quantitatif dès 9-10 ans, âge auquel la quasi totalité des reprises ont un statut d'anaphore. Les premières unités à être dotées d'une fonction anaphorique à l'intérieur d'un texte sont les pronoms. Les unités identiques de point de vue catégoriel mais dotées de statuts différents se côtoient dans un même texte jusqu'à ce que le fonctionnement anaphorique de base soit bien stabilisé, c'est-à-dire vers 9-10 ans.

Lambert (2003), s'intéressant à la cohésion et la connexité dans des récits d'enfants, note que, si à 10 ans, le marquage du statut des entités est systématique, la régularisation du maintien de la référence par la pronominalisation pose encore problème.

Concernant le choix des unités anaphoriques, l'évolution intervient essentiellement lorsque le fonctionnement de base est stabilisé, à partir de 9-10 ans et jusqu'au collège.

Si le développement des anaphores, de la cohésion et de la cohérence dans les textes narratifs a fait l'objet de nombreuses études, peu portent encore sur le développement de ces notions dans d'autres types de textes, notamment les textes expositifs. Ces derniers ont pour fonction d'exposer des théories ou des concepts. Leur structure est moins prédictible et plus variable que celle des textes narratifs, ils sont davantage liés à une maîtrise décontextualisée du langage. On peut donc supposer que l'usage des anaphores sera différent sur les plans quantitatifs et qualitatifs par rapport aux textes narratifs.

Quant à la modalité orale ou écrite, De Weck (1991) s'interroge sur son influence et témoigne de l'intérêt à comparer des textes de même nature produits dans les deux modalités. Les contraintes liées à la production orale et celles liées à la production écrite sont en effet fort différentes. Les marques de cohésion jouent le rôle de témoins de la réorganisation des contenus à produire sur la base des contraintes rhétoriques spécifiques à la production écrite (Favart, 2005 : 32).

\section{Méthodologie}

\subsection{Sujets}

Deux groupes d'enfants (bilingues franco-turcs et monolingues français) âgés en moyenne de 11 ans et scolarisés en école primaire au CM2 composent notre population d'étude. Ils sont constitués de 47 sujets. Le nombre de filles est égal à celui des garçons. Le tableau ci-dessous présente les élèves par population. 


\begin{tabular}{lcc}
\hline Population & Monolingues français & Bilingues franco-turcs \\
\hline Nombre d'élèves & 23 & 24 \\
Age moyen & $11 ; 01$ & $11 ; 00$ \\
Ecarts d'âge & $09 ; 09-11 ; 09$ & $09 ; 07-11 ; 11$ \\
\hline
\end{tabular}

Tableau 1 : Nombre d'élèves par population et niveau scolaire.

Les enfants bilingues sont issus de familles émigrées turques à Rouen de niveau éducatif bas, d'origine socio-économique défavorisée et résidant à Rouen. Ces enfants de la deuxième génération sont tous nés en France. Ils ont acquis la langue turque exclusivement dans le milieu familial jusque l'âge de 7 ans et à partir de cet âge ils ont pu bénéficier de cours de langue turque dans le cadre de l'enseignement de la langue et la culture d'origine (ELCO). Ces classes leur permettent d'acquérir la lecture et l'écriture dans la langue turque, ainsi que des connaissances concernant l'histoire et la géographie de la Turquie. Ils ont commencé à apprendre le français (appelé à devenir leur langue dominante) lors de leur entrée en école maternelle, vers l'âge de 3 ans. Les pères sont majoritairement ouvriers ou maçons, tandis que les mères sont principalement mères au foyer.

Les enfants monolingues français sont issus de familles de faible instruction, d'origine socio-économique défavorisée, qui fréquentent la même école et résident dans les mêmes quartiers que les enfants bilingues. Pour ce groupe, les pères sont employés, techniciens qualifiés, exercent des professions libérales. Les mères sont le plus souvent employées.

\subsection{Collecte, transcription et codage des données}

L'ensemble du protocole, repris de Berman et Verhoeven (2002), comprenait quatre tâches. Avant de les réaliser, les élèves ont d'abord rempli un questionnaire sur leur pratique des activités littéraciées (lecture et écriture en dehors de l'école) et ont ensuite visionné un film de trois minutes. Ce film met en scène des problèmes fréquemment rencontrés à l'école : bagarres entre élèves, élèves qui copient les uns sur les autres, éviction d'un élève par un groupe. Après avoir visionné le film, chacun des sujets est invité à produire 4 textes dans des situations variant du point de vue de deux paramètres : le mode de production (oral ou écrit) et le type textuel (narratif ou expositif). Les sujets bilingues ont produit leurs textes dans les deux langues, d'abord en français et ensuite en turc. Afin d'éviter les reprises systématiques d'une langue à l'autre, pour les bilingues, un espacement d'au moins une semaine avait lieu entre les deux sessions. Par ailleurs, afin de réduire d'éventuels effets de l'ordre de passation, on a adopté deux ordres de passation différents : « oral avant écrit » (ordre A) et «écrit avant oral » (ordre B). Le texte dit narratif est une narration d'expérience personnelle : le sujet doit raconter une histoire à propos d'une bagarre qui lui est arrivée et le texte expositif consiste en l'expression d'une opinion personnelle au sujet de la violence et des incivilités à l'école.

Afin de permettre une comparaison entre les différents textes, selon les variables sujets, type de texte et modalité, nous avons fait les choix de transcription suivants :

- Les textes oraux ont été transcrits en orthographe standard, sans ponctuation, en essayant cependant de respecter les marques de l'oral (élisions, marques d'hésitation, répétitions) et les tours de parole entre l'enfant et l'adulte. Voici un exemple de texte oral transcrit (bilingue, narratif, groupe A) :

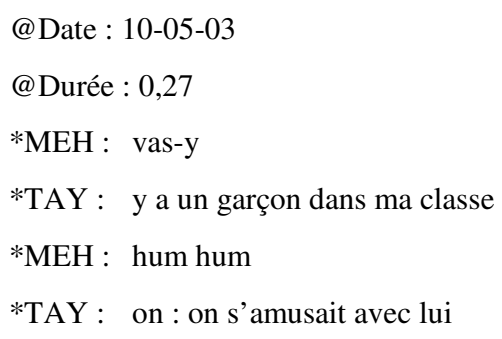


*MEH : hum hum

*TAY : et à un moment \# il m'a il m'a dit un gros mot \# et moi j'étais pas content \# et je lui ai : je lui ai disputé et tout après il m'a il m'a encore insulté après on s'est bagarré

*MEH : hum hum

*TAY : c'est pour ça

*MEH : c'est tout ? d'accord

- Les textes écrits ont été retranscrits tels qu'ils ont été écrits par les enfants, sans correction afin de permettre le comptage des mots. Voici un exemple de texte écrit (monolingue, narratif, ordre B) :

(2) Un jour je suis rentré de l'école est quelqun a appelé il ma dis : je sais que tu à peur pourquoi tu $t$ énferme et je sais que tu et toute seule j'ai tout de suite racrocher. il arrêté pas d'appeler mais je ne reponde plus. Et quand t'il a appelé la dernière fois il a laisser un message sur le répondeur. Quand ma mère et arrivé elle a écouté le message et il avait dit que des bêtise. et un autre jour il a rappeler mon père a sus son numéro de portable c'etait un copain a mon frère et depuis que mon père avait apeler il $m$ 'a jamais rappeler. (Sujet $\mathrm{n}^{\circ} 9$ : Ophélie. NE)

Dans notre démarche d'analyse, nous reprendrons certains éléments de celle utilisée par De Weck (1991). En reprenant sa classification des anaphores, nous nous proposons d'étudier la densité des unités anaphoriques et d'analyser la distribution des anaphores selon la typologie ci-dessous.

I. Les anaphores pronominales :

1. Les pronoms de troisième personne (y compris $y$ et en) : «Je me suis batut avec sésbastien quart il mernever » (Sujet $n^{\circ} 4$, monolingue, ordre A : Julien, NE).

2. Les pronoms démonstratifs (formes simples et composées) : «ca sert à rien » (Sujet $\mathrm{n}^{\circ} 1$, bilingue, ordre A : Fatih, EE).

3. Les pronoms et adjectifs possessifs de troisième personne : «Ma mère devait partir en Turquie pour son père qui était malade » (Sujet $n^{\circ} 9$, bilingue, ordre B : Ümmühan, $\mathrm{NE})$.

4. Les pronoms et adjectifs possessifs de première et de deuxième personne : «Mon ami a dit qu'il n'en avait pas » (Sujet $n^{\circ} 2$, bilingue, ordre A : Turan, NE).

5. Les pronoms relatifs : «Il y en avait qui comme Thibault et Sacha me supportait pour que je la tape $»\left(\right.$ Sujet $n^{\circ} 1$, monolingue, ordre B : David, NE).

6. Les pronoms indéfinis : «Si l'autre fait une erreur... » (Sujet $n^{\circ} 4$, bilingue, ordre A : Bekir, EE).

Les adjectifs possessifs ont une fonction référentielle semblable à celle des pronoms.

II. Les procédés anaphoriques nominaux :

7. La répétition : «Moi je pense que la violence ce n'est pas une chose très bien car : les problèmes ne se règle jamais avec la violence » (Sujet $n^{\circ} 8$, bilingue, ordre $B$ : Müberra, EE).

8. La définitivisation : «un grand à demander...et après le grand a dit...» (Sujet $\mathrm{n}^{\circ} 2$, bilingue, ordre A : Turan, NE).

9. La référenciation déictique intratextuelle: «Ma grave maladie (...) j'avais 6 ans quand j'ai eu cette maladie » (Sujet $\mathrm{n}^{\circ} 11$, ordre B, monolingue: Justine, NE).

10. La substitution lexicale : «j'ai reçus dais coup de pied par un garçon (...) je l'ai dit au maître et il a puni l'élève » (Sujet $n^{\circ} 6$, ordre B, monolingue: Guillaume, NE)

11. La nominalisation : «Etant petite, chez mon tonton, mon grand frère jouait à la pétanque, tout le monde disait de pas passer derrière eux. » (Sujet $n^{\circ} 10$, ordre A, monolingue, Sarah, NE) 
III. La catégorie « autres » :

12. Essentiellement adverbes de lieu et de temps : «Elle me la rendue le lendemain » (Sujet $\mathrm{n}^{\circ}$, ordre B, bilingue: Rüveyda, NE)

Afin de classer les occurrences anaphoriques dans l'une des catégories ci-dessus, nous avons pris comme point de départ la première mention d'un référé. Le calcul de la densité des anaphores permet d'obtenir une évaluation de leur taux d'utilisation selon le type de texte et la modalité, en neutralisant la longueur des productions. Ce calcul consiste en un rapport du nombre d'anaphores sur le nombre de mots des textes.

\section{Les résultats}

\subsection{Les longueurs des textes}

Nous avons mesuré pour chaque texte la longueur en nombre de mots. Berman et Verhoeven (2002) ayant montré que les enfants de 9 ans écrivent moins qu'ils ne parlent, nous émettons l'hypothèse que les textes oraux seront plus longs que les textes écrits, quelque soit la population ou le type de texte. Nous nous attendons à ce que les monolingues et les bilingues produisent des textes de longueur sensiblement identiques, et que les textes narratifs, plus familiers aux sujets et moins coûteux sur le plan cognitif, soient plus longs que les textes expositifs.

Les élèves monolingues produisent des textes narratifs plus longs (88,32 mots en moyenne) que les textes expositifs (67,59 mots en moyenne). Les textes oraux (108,76 mots en moyenne) sont significativement plus longs que les textes écrits (47,15 mots par texte en moyenne). Les élèves bilingues produisent quant à eux des textes expositifs plus longs (87,24 mots en moyenne) que les textes narratifs (71,5 mots en moyenne). Les textes oraux (97,41 mots en moyenne) sont significativement plus longs que les textes écrits (61,33 mots par texte en moyenne). Le tableau 2 précise les données :

\begin{tabular}{|c|c|c|c|c|}
\hline Texte & & & & \\
\hline Modalité & Oral & Ecrit & Oral & Ecrit \\
\hline Population & & Mono & ançais & \\
\hline Nombre total de mots & 2760 & 1303 & 2243 & 866 \\
\hline $\begin{array}{l}\text { Nombre moyen de } \\
\text { mots }\end{array}$ & 120 & 56,52 & 97,52 & 37,65 \\
\hline Ecarts mots par texte & $23-516$ & $14-147$ & $17-346$ & $20-92$ \\
\hline Population & & Biling & o-turcs & \\
\hline Nombre total de mots & 2159 & 1130 & 2322 & 1691 \\
\hline $\begin{array}{l}\text { Nombre moyen de } \\
\text { mots }\end{array}$ & 93,87 & 49,13 & 100,96 & 73,52 \\
\hline Ecarts mots par texte & $36-270$ & $12-102$ & $33-166$ & $21-272$ \\
\hline
\end{tabular}

Tableau 2 : Caractéristiques des textes produits par les élèves monolingues et bilingues selon le type de texte et la modalité.

Il apparaît dans ce tableau que, si les sujets monolingues produisent des textes narratifs plus longs que les textes expositifs, et ce quelle que soit la modalité, il existe une certaine homogénéité dans le nombre de mots dans les textes les plus courts, mais une grande disparité dans le nombre de mots des textes les plus longs. Si les sujets bilingues produisent des textes expositifs plus longs que les textes narratifs, et ce quelle que soit la modalité, on note une certaine disparité également dans les nombres de mots dans les textes les plus courts et les plus longs.

Lorsque nous analysons les textes selon la variable «ordre de passation », chez les élèves monolingues, une grande hétérogénéité est observée, avec toutefois une supériorité plus ou moins marquée du nombre 
moyen de mots par texte dans l'ordre A dans trois des quatre types de textes. Si l'on peut considérer que l'ordre de passation n'a que peu d'influence sur la production des textes narratifs oraux, il n'en va pas de même pour les autres types de textes.

Il existe ainsi un effet très net de l'ordre de passation concernant les textes expositifs oraux, avec un rapport du simple au double entre les textes expositifs oraux produits dans l'ordre B (65,75 mots en moyenne) et ceux produits dans l'ordre A (132,18 mots en moyenne): autrement dit, les sujets monolingues produisent des textes expositifs oraux plus longs lorsqu'ils commencent par la modalité orale que lorsqu'ils produisent le texte oral après avoir rédigé le texte écrit. Nous émettons l'hypothèse que le passage préalable par l'écrit produirait un effet de «brouillon de l'oral» et que dans ce cas, les sujets reprendraient davantage à l'oral le contenu de l'écrit en le développant quelque peu, leurs textes contiendraient alors moins de répétitions et de marques d'hésitations.

Pour les élèves bilingues, de nouveau en évidence une grande hétérogénéité est observée. Ainsi, l'ordre de passation semble avoir assez peu d'influence sur la production des textes narratifs écrits et expositifs oraux, même si on peut noter une légère dominance de l'ordre A pour les textes narratifs et de l'ordre $\mathrm{B}$ pour les textes expositifs. Par ailleurs, nous notons un effet de l'ordre de passation en faveur de l'ordre A (oral puis écrit) dans les textes narratifs oraux et en faveur de l'ordre B (écrit puis oral) dans les textes expositifs écrits.

Les deux populations ne semblent pas réagir de la même manière à l'ordre de passation. Est-il possible d'affiner ces comparaisons? Nous allons tenter de le faire au moyen du tableau 3 :

\begin{tabular}{lcccc}
\hline Population & \multicolumn{2}{c}{ Monolingues français } & \multicolumn{2}{c}{ Bilingues franco-turcs } \\
Modalité & Oral & Ecrit & Oral & Ecrit \\
\hline Ordre A & 128,14 & 87,36 & 101,25 & 52,9 \\
Ordre B & 91 & 100,67 & 94,46 & 67,81 \\
\hline
\end{tabular}

Tableau 3: Nombre moyen de mots selon la modalité et l'ordre de passation dans les textes produits par les élèves monolingues et bilingues.

L'analyse de ce tableau met en évidence un effet de primauté : les textes oraux sont plus longs dans l'ordre A que dans l'ordre B, quelque soit la population, tandis que les textes écrits sont plus longs dans l'ordre B que dans l'ordre A, quelque soit ici encore la population. Les sujets monolingues et les sujets bilingues sont donc tous sensibles à l'effet de primauté. Nous remarquons toutefois que l'effet est plus marqué pour les sujets monolingues que pour les sujets bilingues.

L'analyse du contenu des textes apporte en effet un éclairage sur ces résultats. Alors que les enfants monolingues ont produit des textes expositifs concernant le thème de la violence de manière générale, les enfants bilingues franco-turcs ont davantage eu recours au support du film visionné. Nombre de leurs textes expositifs comportent ainsi des épisodes au cours desquels les enfants bilingues reprennent les situations observées dans le film afin d'argumenter. Ils rappellent les unes après les autres les situations du film en y adjoignant un commentaire.

\subsection{Densité anaphorique}

Nous nous attendons à trouver une plus grande densité anaphorique dans les textes narratifs que dans les textes expositifs, et ce quelque soit la population envisagée. En effet, le fonctionnement anaphorique est maîtrisé plus tôt pour les textes narratifs que pour les autres types de textes, certains n'étant maîtrisés, comme nous l'avons vu précédemment, que vers 14 ans (De Weck, 1991).

Nous émettons par ailleurs l'hypothèse qu'en raison des contraintes liées à l'écrit, les textes produits dans cette modalité présenteront une plus grande densité anaphorique.

Nous pensons que les textes des sujets monolingues et ceux des sujets bilingues auront des densités anaphoriques comparables. 
Pour calculer la densité anaphorique, nous avons rapporté le nombre d'anaphores au nombre de mots dans les textes. Le tableau 4 présente les résultats de la densité anaphorique.

\begin{tabular}{lcccccccc}
\hline $\begin{array}{l}\text { Type de } \\
\text { texte }\end{array}$ & \multicolumn{3}{c}{ Narratif } & \multicolumn{3}{c}{ Expositif } \\
Modalité & \multicolumn{2}{c}{ Oral } & \multicolumn{2}{c}{ Ecrit } & \multicolumn{2}{c}{ Oral } & \multicolumn{2}{c}{ Ecrit } \\
Population & Mono. & Bil. & Mono. & Bil. & Mono. & Bil. & Mono. & Bil. \\
\hline Ordre A & 17 & 17,03 & 13,83 & 14,94 & 12,1 & 18,1 & 7,91 & 13,81 \\
Ordre B & 15,2 & 12,57 & 9,95 & 9,54 & 13,69 & 21,23 & 5,6 & 11,6 \\
\hline
\end{tabular}

Tableau 4 : Densité anaphorique (pourcentage d'anaphores par rapport au nombre de mots dans les textes produits) dans les textes produits selon l'ordre de passation et la modalité.

En comparant les densités anaphoriques des deux groupes selon l'ordre de passation, nous constatons que les deux populations se comportent de la même manière, à savoir qu'elles font montre d'une densité anaphorique supérieure dans l'ordre A pour les textes narratifs oraux et écrits et pour les textes expositifs écrits, ainsi que d'une densité anaphorique supérieure dans l'ordre B pour les textes expositifs oraux. Les écarts de densité entre les deux ordres sont toutefois un peu plus marqués chez les sujets bilingues.

Les deux points précédents mettent en évidence un comportement similaire des deux populations quant aux effets liés à l'ordre de passation et à la modalité orale ou écrite. Concernant ce dernier point, nous émettons l'hypothèse que la longueur des textes oraux nécessite de la part des sujets un recours plus important aux anaphores pour maintenir la cohésion de leurs textes.

Par contre, il est apparu que les sujets monolingues et bilingues ont un comportement différent quant au type de texte, les monolingues produisant des textes narratifs plus denses en anaphores que les textes expositifs, tandis que les sujets bilingues produisent des textes expositifs plus riches en anaphores que les textes narratifs.

\subsection{Densité selon le type d'anaphore utilisé}

Afin d'approfondir notre étude des anaphores, nous envisageons maintenant la répartition des types d'anaphores en pourcentage par rapport au nombre total d'anaphores, et ce pour chaque type de texte et chaque population. Nos hypothèses sont les suivantes :

- Hypothèse 1 : pour les textes narratifs, les anaphores pronominales seront les plus nombreuses, suivies des syntagmes nominaux. En raison de la disjonction par rapport au référent et du mode discursif autonome, les pronoms personnels de troisième personne, les pronoms et adjectifs possessifs de troisième personne et les relatifs devraient être plus nombreux.

- Hypothèse 2 : concernant les textes expositifs, les anaphores pronominales seront également les plus nombreuses, suivies des anaphores nominales et de la catégorie autre.

- Hypothèse 3 : la comparaison des textes expositifs et narratifs devrait mettre en évidence une différence qualitative et quantitative des types d'anaphores, les textes expositifs marquant notamment un effacement de l'énonciateur. Les pronoms et adjectifs possessifs de première et de deuxième personne devraient être moins nombreux.

- Hypothèse 4 : les différents types d'anaphores devraient être représentés de la même façon (en terme de pourcentage) dans les textes des monolingues et des bilingues.

Le tableau 5 présente les pourcentages obtenus pour chaque catégorie d'anaphores en fonction du type de texte et de la population monolingue ou bilingue. 


\begin{tabular}{|c|c|c|c|c|}
\hline \multirow{2}{*}{$\begin{array}{l}\text { Texte } \\
\text { Modalité }\end{array}$} & \multicolumn{2}{|c|}{ Narratif } & \multicolumn{2}{|c|}{ Expositif } \\
\hline & Oral & Ecrit & Oral & Ecrit \\
\hline Population & \multicolumn{4}{|c|}{ Monolingues français } \\
\hline $\begin{array}{l}\text { Anaphores } \\
\text { pronominales }\end{array}$ & 13,4 & 9,8 & 11,4 & 6,1 \\
\hline Anaphores nominales & 2,6 & 1,5 & 1,3 & 0,6 \\
\hline Autres & 0,04 & 0,08 & 0 & 0,12 \\
\hline Population & \multicolumn{4}{|c|}{ Bilingues franco-turcs } \\
\hline $\begin{array}{l}\text { Anaphores } \\
\text { pronominales }\end{array}$ & 12,7 & 9,5 & 14,7 & 10,7 \\
\hline Anaphores nominales & 2 & 2,6 & 0,95 & 1,23 \\
\hline Autres & 0,14 & 0 & 0 & 0,06 \\
\hline
\end{tabular}

Tableau 5: Répartition (en \% du nombre de mots) des types d'anaphores dans les textes produits par les élèves monolingues français et bilingues franco-turcs.

Dans les deux types de textes et les deux modalités, qu'il s'agisse des productions des monolingues ou des bilingues, les anaphores pronominales sont les plus représentées, nos hypothèses 1 et 2 sont donc vérifiées.

Comme nous en avions également émis l'hypothèse, les pourcentages sont relativement proches entre les populations monolingue et bilingue, avec toutefois de légères différences en faveur des bilingues concernant les anaphores pronominales dans les textes expositifs et les anaphores nominales à l'écrit et en faveur des monolingues concernant les anaphores pronominales dans les narrations, les anaphores nominales à l'oral et la catégorie autres.

Concernant l'hypothèse 3 , nous retrouvons effectivement des différences quantitatives et qualitatives entre les textes narratifs et expositifs :

- Les textes expositifs contiennent un peu plus d'anaphores pronominales que les textes narratifs, chez les sujets bilingues, en modalité orale ou écrite, tandis que les textes narratifs contiennent plus d'anaphores pronominales que les textes expositifs chez les sujets monolingues, quelle que soit la modalité. Cette différence est-elle à attribuer à l'effet de longueur des textes ou à une différence de traitement des textes et donc des anaphores, c'est une question qu'une analyse qualitative devrait nous permettre d'éclairer.

- Les textes narratifs contiennent davantage d'anaphores nominales que les textes expositifs.

- Les résultats sont plus contrastés pour ce qui concerne la catégorie autre, les densités étant très faibles. Les densités sont les plus importantes pour les textes narratifs oraux et les textes expositifs écrits. Il est toutefois difficile d'interpréter ces résultats, étant donné le très faible nombre d'anaphores et de textes concernés.

\subsection{Répartition qualitative des anaphores}

Une étude qualitative des types d'anaphores utilisés par les sujets monolingues et bilingues nous permet d'affiner encore nos comparaisons entre ces deux populations et de préciser l'incidence du type de texte et de la modalité.

La comparaison des densités des anaphores pronominales met en évidence les hiérarchies suivantes :

Narration orale : Pronoms personnels $3^{\text {ème }}$ personne $>$ pronoms démonstratifs $>$ Pronoms et adjectifs possessifs de $1^{\text {ère }}$ et $2^{\text {ème }}$ personne $>$ relatifs $>$ Pronoms et adjectifs possessifs de $3^{\text {ème }}$ personne $>$ indéfinis. Cette hiérarchie est la même pour les sujets bilingues et monolingues.

Narration écrite : Pronoms personnels $3^{\text {ème }}$ personne> Pronoms et adjectifs possessifs de 1 ère et $2^{\text {ème }}$ personne $>$ pronoms démonstratifs tant chez les sujets monolingues que chez les sujets bilingues. La 
hiérarchie est différente pour les pronoms possessifs de $3^{\text {ème }}$ personne et les pronoms relatifs, les indéfinis étant quant à eux les moins représentés dans les deux populations.

Nous notons que les pronoms démonstratifs sont plus présents dans les narrations orales que dans les narrations écrites. Nous émettons l'hypothèse que la différenciation entre les registres de l'oral et de l'écrit ainsi que la différence de longueur des textes peuvent expliquer cette différence.

L'importance des pronoms personnels de $3^{\text {ème }}$ personne valide notre première hypothèse. Cependant, les pronoms et adjectifs possessifs de 1 ère et de $2^{\text {ème }}$ personne sont bien plus nombreux que ceux de $3^{\text {ème }}$ personne, contrairement à nos attentes, de même que les relatifs n'apparaissent qu'en quatrième place. Les récits produits ici étant des récits d'expérience personnelle, les pronoms personnels de 1 ère personne sont nombreux et par conséquent les pronoms et adjectifs possessifs de $1^{\text {ère }}$ et $2^{\text {ème }}$ personne le sont également.

Les relatifs sont peu utilisés, ainsi que les indéfinis.

Exposition orale: Démonstratifs $>$ Pronoms personnels $3^{\text {ème }}$ personne $>$ relatifs pour nos deux populations.

Exposition écrite : Démonstratifs $>$ Pronoms personnels $3^{\text {ème }}$ personne pour les sujets monolingues et bilingues. Dans les textes des sujets monolingues viennent ensuite les indéfinis, puis les relatifs, les possessifs ( $1^{\text {ère }}, 2^{\text {ème }}$ et $3^{\text {ème }}$ ) étant quant à eux totalement absents, tandis que dans les textes des sujets bilingues, la suite de la hiérarchie est la suivante: relatifs > pronoms possessifs de 3 ème personne>indéfinis>pronoms possessifs de $1^{\text {ère }}$ et 2 ème personne.

Les pronoms démonstratifs sont les plus utilisés dans les textes expositifs oraux et écrits. Ils servent en effet souvent à introduire un jugement après avoir développé un exemple :

(3) Dans ce film il y avait beaucoup de violance. Ce n'est pas bien de recopier sur le camarade parce que si l'autre a faut lui assi a tout faut. ce n'est pas bien de se bagaré quand il y a une dame qui perd un billet et que la fille elle le ramase se n'est pas du tout bien. Et que un garçon dit bonjour a ses amis et qui n'en dit pas a un garcon sa ma ra pas plus. (Sujet $n^{\circ} 8$ : Meliha. EE)

Quant aux pronoms personnels de troisième personne, leur densité est à rapprocher du contenu des textes expositifs où les sujets ont recours à des exemples indéfinis «un camarade, un ami » repris par les pronoms personnels de $3^{\text {ème }}$ personne.

L'analyse qualitative vient donc modérer les différences notées quant à la densité des anaphores pronominales entre nos deux populations.

Les anaphores nominales constituent la deuxième catégorie d'anaphores en densité dans les textes produits par les sujets. Elles sont beaucoup moins nombreuses que les anaphores pronominales et l'analyse qualitative se base sur de très faibles densités.

Les densités des anaphores nominales sont plus contrastées entre les deux populations. Toutefois, il n'est question ici que de peu d'anaphores, les résultats sont donc à prendre avec précaution.

Concernant les narrations orales et écrites, les répétitions sont les plus nombreuses, tant chez les sujets monolingues et les bilingues. La répétition semble en effet de l'anaphore nominale la plus simple à mettre en œuvre.

Les répétitions sont également les plus nombreuses dans les textes expositifs oraux et écrits, suivies des définitivisations. 
Enfin, nous notons que les répétitions sont plus nombreuses dans les textes narratifs que dans les textes expositifs. Nous attribuons cette différence à la longueur des textes et à leur contenu (multiplicité des personnages).

\section{Conclusion}

La réussite scolaire nécessite la maîtrise de compétences langagières particulières, tant à l'écrit qu'à l'oral, compétences liées à des situations souvent décontextualisées et faisant appel à un haut niveau d'exigence cognitif. Les anaphores, de par leur définition, nous semblent être un bon indicateur d'un niveau de maîtrise langagière compatible avec les exigences scolaires.

Comme nous l'avons vu, les études concernant les anaphores ont essentiellement porté sur les textes narratifs. Or, les textes produits dans le cadre scolaire au niveau primaire sont tout autant des narrations que des textes expositifs ou argumentatifs. Il nous a donc paru judicieux de comparer l'usage des anaphores dans ces deux types de textes.

L'étude des textes produits par les enfants montre des productions différentes au niveau de la longueur des textes : si les sujets monolingues produisent comme nous l'attendions des textes narratifs plus longs que les textes expositifs, le constat est inverse dans la population bilingue. Plusieurs hypothèses peuvent venir expliquer cette différence. L'éventualité d'un biais méthodologique n'est pas à exclure, les sujets bilingues ayant suivi deux fois le protocole, une fois en français, une fois en turc. Une différence de fonctionnement cognitif (plus analytique chez les bilingues ?) et de familiarité avec les narrations pourrait également expliquer ces différences. Il conviendrait pour infirmer ou confirmer ces hypothèses d'élaborer un autre protocole.

Nos résultats portant sur la densité anaphorique montrent chez les sujets bilingues franco-turcs une densité anaphorique globale très légèrement supérieure à celle obtenue pour l'ensemble des textes par les sujets monolingues. Les deux populations se comportent par ailleurs de manière similaire quant aux effets liés à l'ordre de passation ou la modalité. Une différence apparaît par contre en fonction du type de texte, les textes narratifs présentant une densité anaphorique plus élevée chez les sujets monolingues, tandis que la densité anaphorique est supérieure chez les bilingues franco-turcs dans les textes expositifs. Nous relativisons toutefois cette dernière conclusion en raison des caractéristiques des textes expositifs écrits par les sujets bilingues.

Nos résultats tendent ainsi à confirmer l'absence de différence dans les productions en français des enfants monolingues français et bilingues franco-turcs : les enfants de même milieu socio-culturel ont des comportements langagiers d'utilisation des anaphores similaires, qu'ils soient monolingues ou bilingues. En ce sens, nos résultats vont à l'encontre de nombre d'idées reçues qui continuent d'avoir cours aujourd'hui sur le bilinguisme des enfants issus de l'immigration dans les écoles françaises.

\section{Références bibliographiques}

Benveniste, E. (1966). Problèmes de linguistique générale. Paris : Gallimard.

Berman, R.A. \& Slobin, D.I. (1994). Relating events in narrative: A crosslinguistic developmental study. Hillsdale, NJ : L. Erlbaum Associates.

Berman, R.A. \& Verhoeven, L (2002). «Cross-linguistic perspectives on the develoment of text-production abilities: speech and writing», Written Language \& Literacy, 5:1, 1-43.

Bronckart, J.-P. (1997). Activité langagière, textes et discours. Pour un interactionisme socio-discursif. LausanneParis : Delachaux et Niestlé.

Charolles, M. (1978) «Introduction aux problèmes de la cohérence des textes », Langue Française, 38, 7-41.

Chastain, C. (1975). «Reference and Context», in Gunderson, K. (ed), Language Mind and Knowledge. Minneapolis : University of Minnesota Press, 194-269. 
Corblin, F. (1995). Les formes de reprise dans le discours. Anaphores et chaînes de référence. Rennes: Presses Universitaires de Rennes.

Corblin, F. (2005). «Les chaînes de la conversation et les autres », in Gouvard, J.-M (dir.), De la langue au style. Lyon : Presses Universitaires de Lyon, 233-254.

Courtès, J. (1991). Analyse Sémiotique du Discours, de l'énoncé à l'énonciation. Paris : Hachette Supérieur.

Favart, M. (2005). «Les marques de cohésion », Psychologie Française, 50/3 : 305-322.

Fox, B. (1987). Discourse structure and anaphora: Written and conversational English.Cambridge : Cambridge University Press.

Halliday, M.A.K. \& Hasan, R. (1976). Cohesion in English. Londres : Longman.

Haroche, C. \& Pécheux, M. (1972). «Manuel pour l'utilisation de la méthode d'analyse automatique du discours (AAD) », TA Informations, 13(1), 13-55.

Hickmann, M. (2001). «Le développement de la cohésion discursive : le récit à l'oral chez l'enfant », Langage et Pratique, 27, 43-52.

Hickmann, M. (2004). «Coherence, Cohesion, and Context. Some Comparative Perspectives in Narrative Development », in Strömqvist, S. \& Verhoeven (eds), Relating events in narrative : Typological and contextual perspectives. Mahwah, NJ: Lawrence Erlbaum Associates, Volume 2, 281-306.

Jakobson, R, (1963). Essais de linguistique générale. Paris, Minuit.

Karmiloff, K. \& Karmiloff-Smith, A., (2003). Comment les enfants entrent dans le langage. Paris : Retz (1ère éd., 2001).

Karmiloff-Smith, A. (1996). Beyond Modularity: A developmental perspective on cognitive science. CambridgeLondon: MIT Press.

Kerbrat-Orecchioni, C. (1999). L'énonciation. Paris : Armand Colin (4ème éd.).

Kleiber, G. (2001). L'anaphore associative. Paris : PUF.

Lambert, M. (2003). «Cohésion et connexité dans des récits d'enfants et d'apprenants polonophones du français », Marges linguistiques, 5, (http://www.marges-linguistiques.com), 106-121.

Lyons, J. (1980). Sémantique linguistique. Paris : Larousse (1ère éd. 1978).

Mayer, M. (1969). Frog, where are you?. New York : Dial Books for Young Readers.

Milner, J.C. (1982). Ordres et raisons de langue. Paris : Seuil.

Riegel, M., Pellat, J.C. \& Rioux, R. (1994). Grammaire méthodique du français. Paris : PUF.

Salazar-Orvig, A., Fayolle V., Hassan R., Leber-Marin J., Marcos H. Morgenstern A., Pares J. (2004). « Emergence des marqueurs anaphoriques : le cas des pronoms », Cahiers d'Acquisition du Langage et Pathologie, 24, 57-82.

Weck (de), G. (1991). La cohésion dans les textes d'enfants. Etude du développement des processus anaphoriques. Lausanne-Paris : Delachaux et Niestlé. 